\title{
Pattern-Dependent Charging in Plasmas: Electron Temperature Effects
}

\author{
Gyeong S. Hwang and Konstantinos P. Giapis* \\ Division of Chemistry and Chemical Engineering, California Institute of Technology, Pasadena, California 91125
}

(Received 5 December 1996; revised manuscript received 18 February 1997)

\begin{abstract}
The differential charging of high-aspect-ratio dense structures during plasma etching is studied by two-dimensional Monte Carlo simulations. Enhanced electron shadowing at large electron temperatures is found to reduce the electron current density to the bottom of narrow trenches, causing buildup of large charging potentials on dielectric surfaces. These potentials alter the local ion dynamics, increase the flux of deflected ions towards the sidewalls, and result in distorted profiles. The simulation results capture reported experimental trends and reveal the physics of charging damage. [S0031-9007(97)03724-1]
\end{abstract}

PACS numbers: $52.75 . \mathrm{Rx}$

Patterned surfaces are frequently exposed to plasmas as in measurements of plasma parameters through minuscule sampling orifices or in applications ranging from etching, deposition, and ashing in microelectronics, to selective surface functionalization [1]. When the patterns consist of insulating materials, plasma-induced charging may cause ion deflection with undesirable consequences; for example, mask charging leads to sidewall profile irregularities when etching high-aspect-ratio features [2]. In addition, orifice sidewall charging may distort the measurement of flux, energy, and angular distributions of ions [1,3]. Pattern-dependent charging is at the heart of a multitude of fascinating new phenomena recently observed in semiconductor processing [4-6], whose fundamental physics is not understood.

Charging effects are perhaps nowhere more apparent than when plasma etching dense submicron patterns of transistors for computer memories [4-6]. These structures involve insulating (mask, gate oxide) and conductive (polysilicon or metal gate) materials in various geometric arrangements. Differential microstructure charging results when the sidewalls limit the flux of the isotropic electrons to the trench bottom (electron shading [4]), a situation that allows more electrons to accumulate at the mask sidewalls while more ions accumulate at the trench bottom $\left(\mathrm{SiO}_{2}\right)$. Charge builds up until the bottom surface acquires a potential large enough to repel a sufficient number of ions so that the ion and electron currents to the bottom surface balance $[7,8]$. The repelled ions bombard the sidewalls, increasing the charging potential of the poly-Si lines. As the potential difference across the underlying gate oxide builds up, large Fowler-Nordheim tunneling currents are possible, leading to oxide degradation and breakdown [4]. Further, profile distortion becomes unavoidable when the deflected ions acquire translational energy high enough for reaction at the sidewalls [8]. This possibility was manifested by the "notching" effect [5,6], describing a wedgelike sidewall profile irregularity whose typical location and shape are depicted in Fig. 1. Pattern-dependent charging effects have puzzled the etching community for many years; their damaging consequences are feared to impede progress towards smaller logic devices for faster microprocessors and denser memories.

Understanding charging damage requires detailed modeling and simulation of ion and electron dynamics in plasmas, through sheaths, and as they impinge at various microstructure surfaces where they cause charging, physical sputtering and chemical reactions, or undergo inelastic scattering. A predictive feature-scale simulation that discusses these steps and simplified models to describe them has been presented elsewhere [8]. In this Letter, we report on the physics of charging in high-aspect-ratio (4:1) structures and examine the influence of the electron temperature $\left(T_{e}\right)$, a parameter that has been speculated to control notching in cw plasmas [6,9]. Previous attempts to model pattern-dependent charging and the influence of $T_{e}$ were based on nonphysical redistribution of charge on conductive surfaces [10], resulting in unrealistic potential distributions and predictions that contradict experimental results.

Typical conditions for modern high-density plasma reactors [11] are assumed: low pressure ( $<10 \mathrm{mTorr})$, uniform $\mathrm{Cl}_{2}$ plasma of density $1 \times 10^{12} \mathrm{~cm}^{-3}$, dissociated to a degree that renders etching ion limited. The sheath voltage is $V_{\mathrm{sh}}=0.5 V_{\mathrm{rf}}(1+\sin \omega t)+V_{\mathrm{dc}}[12]$, where $V_{\mathrm{rf}}=60 \mathrm{~V}$ is the $\mathrm{rf}$ bias, $V_{\mathrm{dc}}$ is the minimum dc sheath voltage, and $\omega=400 \mathrm{kHz}$ is the rf bias frequency. The ion temperature is taken to be $0.5 \mathrm{eV}$. For simulation purposes, we will consider three electron temperatures: 2,4 , and $8 \mathrm{eV}$,

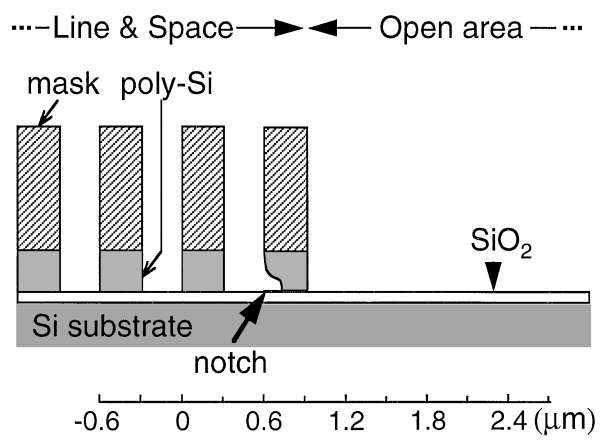

FIG. 1. Schematic depiction of the line-and-space structure considered in the simulation. The location of the notch is also shown. The open area is assumed to be infinite. 
assumed to be achievable without altering plasma density. However, $V_{\mathrm{dc}}$ must change to sustain a realistic sheath; an approximate value can be determined by assuming $V_{\mathrm{dc}} \propto T_{e}$ [12]. Using the experimental value of $V_{\mathrm{dc}}=$ $7 \mathrm{~V}$ at $T_{e}=4 \mathrm{eV}$ [5], we obtain $V_{\mathrm{dc}}=3.5$ and $14 \mathrm{~V}$ at $T_{e}=2$ and $8 \mathrm{eV}$, respectively [13].

The pattern of Fig. 1 borders a widely open area and consists of isolated $0.3 \mu \mathrm{m}$ lines separated by $0.3 \mu \mathrm{m}$ spaces (trenches). At the onset of overetching [14], each feature consists of $0.9 \mu \mathrm{m}$ photoresist mask (insulating) onto $0.3 \mu \mathrm{m} n^{+}$-poly-Si gates, formed on top of a uniform layer of $\mathrm{SiO}_{2}$, thick enough $(>100 \mathrm{~nm})$ to prevent tunneling currents to the $\mathrm{Si}$ substrate; thus, the simulation targets worst-case charging effects.

Pattern charging is performed by a Monte Carlo simulation of ion and electron transport first through a collisionless sheath and then through the microstructure spaces, as described elsewhere [8]. Briefly, realistic ion and electron energy and angular distributions at the wafer are calculated from sheath theory [11] for each $T_{e}$. Then, charged particles are followed as they impinge at various surfaces, where they transfer their charge. Charge deposition creates local electric fields determined by solving the Laplace equation. The fields are used to alter ion trajectories and are modified self-consistently as more charge accumulates. Steady-state charging is reached when the potential distribution along the bottom $\mathrm{SiO}_{2}$ surface no longer changes [8]. Surface currents and secondary electron emission are both neglected.

A larger $T_{e}$ results in a larger Bohm velocity for ions entering the sheath. The latter causes the low energy peak of the bimodal ion energy distribution at the wafer to shift to higher energies [15]. A larger $T_{e}$ also results in more energetic electrons impinging onto the microstructure. Given the relatively isotropic electron angular distribution at the wafer [8], higher energy electrons will cause more negative charge accumulation at the upper sidewalls. Simultaneously, the higher energy ions contribute less to neutralizing this charge. The entrance potential becomes more negative, thus, impeding more electrons from entering the trench. Indeed, as $T_{e}$ is increased from 2 to $8 \mathrm{eV}$, the steady-state electron current to the bottom $\mathrm{SiO}_{2}$ surface of the last trench is calculated to decrease by $33 \%$. Recently, Kamata and Arimoto [16] verified the reduction in electron current through a dielectric structure with aspect ratio of 1.6 following an increase in electron temperature. Further, the potential at the upper photoresist sidewall of the edge line facing the open area becomes also more negative at larger $T_{e}$; this potential is now felt farther out in the open area, thus deflecting a number of electrons that approach closer to the edge line and would have been attracted to the outer poly-Si sidewall at lower $T_{e}$. This apparent reduction in accumulating electrons is counterbalanced by an increase in the number of electrons attracted from the open area as a result of the larger equipotential of the poly-Si edge line at the larger $T_{e}$ (vide infra).
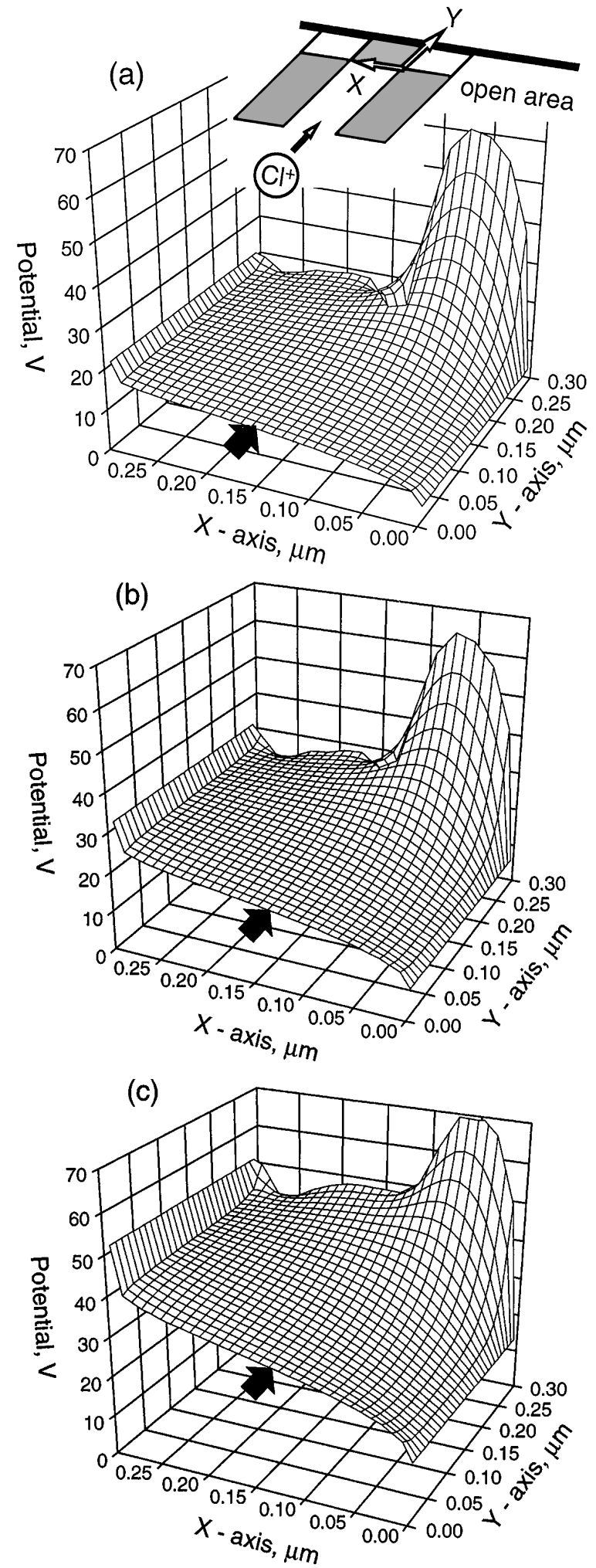

FIG. 2. Three-dimensional charging potential distributions in the trench area bordered by the poly-Si sidewalls for electron temperatures of (a) $2 \mathrm{eV}$, (b) $4 \mathrm{eV}$, and (c) $8 \mathrm{eV}$. The other plasma parameters were as described in the text. The inset illustrates the area of interest and defines the origin for the potential surface. The microstructure has been rotated to allow for a more convenient description of ion motion in the trench. The arrows show the direction of ions as they approach the potential surface. 
The potential distribution in the trench next to the edge line, plotted in Fig. 2, reveals the perturbation in the local ion dynamics occurring as a result of surface charging. Gradients on this potential surface are a measure of the electric field that influences ion motion. As previously shown [8], an asymmetric potential distribution, with a pronounced peak near the inner sidewall foot of the edge line, is critical for notch formation. The peak value depends weakly on $T_{e}$ and strongly on the $\mathrm{rf}$ bias and frequency. However, the charging potential distribution in the trench space away from the peak increases significantly with $T_{e}$. For example, the potential at the center of the bottom surface increases from about 18 to $48 \mathrm{~V}$, when $T_{e}$ is raised from 2 to $8 \mathrm{eV}$, respectively. This behavior is a consequence of the increased entrance barrier to electrons at larger $T_{e}$. The positive potential at the trench bottom must increase to deflect more ions, so that the ion and electron currents to the bottom surface can be balanced. More deflected ions to the poly-Si sidewalls cause the corresponding potentials to increase. The change in boundary potentials is such that the tilt of the potential surface in the trench space towards the outermost line actually increases. Thus, more ions will be deflected towards the inner side of the outermost polySi line and these ions should acquire a larger translational energy as they fall down a steeper potential gradient. It becomes obvious now why profile distortion and notching will worsen with increasing $T_{e}$ [6].

The calculated ion and electron fluxes to both sidewalls of the poly-Si edge line are quantified in Fig. 3. Notice that the ion flux to the outer sidewall and electron flux to the inner sidewall are insensitive to $T_{e}$. Thus, the increase in ion current to the inner sidewall at larger $T_{e}$ is balanced by an equal increase in the electron current to the outer sidewall. The latter is not caused by greater "electron irradiation" of the outer sidewall at larger $T_{e}$ [6]; it rather is a result of the increase in the poly-Si equipotential

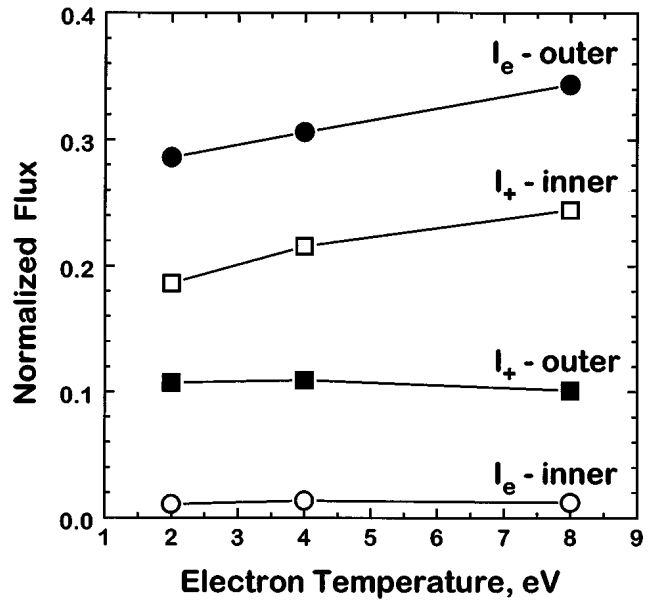

FIG. 3. The normalized ion $\left(I_{+}\right)$and electron $\left(I_{e}\right)$ fluxes to the inner (open symbols) and outer (filled symbols) poly-Si sidewalls of the edge line after steady-state charging has been reached, as a function of the electron temperature. that follows the enhanced ion accumulation at the inner sidewall. Figure 4 demonstrates how the steady-state potentials of the edge line and its neighbor change with electron temperature. Both potentials increase, albeit not at the same rate. Their difference - a measure of the electric field that accelerates ions towards the sidewall as they traverse the trench-increases with $T_{e}$, suggesting that the deflected ions should become more energetic. However, the average ion energy was calculated not to increase as much as the potential difference would predict, a result of the considerable increase in the number of deflected lower energy ions that pin the average energy down. Therefore, a significant notch widening and deepening is predicted to occur at larger $T_{e}$ as a consequence of the increase in the flux of deflected ions, rather than the translational energy acquired by these ions.

The steady-state charging potentials are needed to calculate the trajectories of ions as they get deflected from, scatter onto, and react with the microstructure surfaces during the profile evolution simulation, performed as described before [8]. As etching of the sidewall proceeds, charging of the exposed $\mathrm{SiO}_{2}$ leads to significant forward deflection of energetic ions which contribute to notching. Thus, the etching simulation becomes coupled to transient charging in the etched area, further complicating the calculation. The problem becomes tractable by solving for a new potential distribution every time the notch apex is advanced. Poly-Si etching is assumed to be ion limited while spontaneous etching is neglected. Material is removed from the surface by sputtering of $\mathrm{SiCl}_{x}$ moieties with an energy and angular dependent etch yield [8]. Only single-bounce scattering on the Cl-covered $\mathrm{SiO}_{2}$ is included as all of the scattered atoms impinge subsequently on poly-Si [17].

Profile evolution simulations have been performed for the three electron temperatures, and the profiles obtained for (an estimated) 100\% overetching time [18] are shown in Fig. 5. As expected, increasing $T_{e}$ worsens notching.

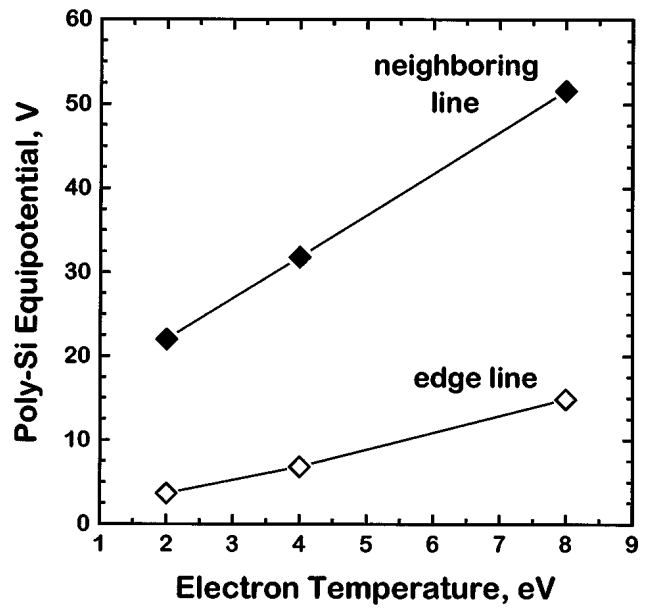

FIG. 4. The steady-state equipotentials of the poly-Si edge line and its neighbor as a function of the electron temperature. 

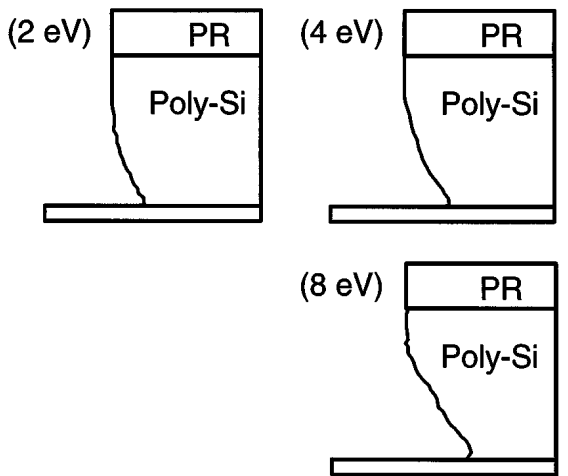

FIG. 5. Notch profiles of the inner sidewall of the edge line, predicted after fixed overetching for three electron temperatures, as indicated. The other plasma parameters were as described in the text. The poly-Si aspect ratio has been preserved; however, the photoresist (PR) vertical dimension has been truncated to save space.

The profile for $T_{e}=8 \mathrm{eV}$ exhibits the deepest and widest notch with upper sidewall recession, a consequence of the increased flux in deflected energetic ions towards the upper poly-Si sidewall. The profile for $T_{e}=4 \mathrm{eV}$ shows a narrower notch, more typical of the plasma conditions employed [5]. Remarkably, notching persists even for $T_{e}=2 \mathrm{eV}$; since a lower $T_{e}$ may not be possible for high-density plasmas [11], this observation suggests that decreasing $T_{e}$ can help reduce but not completely eliminate notching. These results are in good agreement with trends observed in the experiments of Fujiwara et al. [6].

In conclusion, plasma-induced charging of patterned surfaces, and the concomitant perturbation of the ion and electron dynamics in the pattern spaces, is critically influenced by the electron temperature. When etching dense patterns of polysilicon-on-insulator structures, increased bottom surface charging at larger $T_{e}$ causes more ions to be deflected and accelerated towards the sidewalls where they etch, thus distorting the profile. The polysilicon potentials also increase; when the electric field that forms across the underlying gate oxide exceeds the oxide breakdown threshold $(8-12 \mathrm{MV} / \mathrm{cm})$, tunneling currents will flow causing oxide failure. Notching can be reduced but not completely eliminated by just decreasing $T_{e}$. However, operation at lower electron temperatures should be preferred in view of the reduction in the gate potentials. The electron temperature can be reduced by increasing the plasma pressure and/or the effective plasma size [11]. Thus, the enlarged plasma chambers required for uniform etching of $300 \mathrm{~mm}$ wafers could have the added benefit of low $T_{e}$ operation. Further reduction in notching should be possible by combining low $T_{e}$ etching with a simultaneous change in the etch chemistry [8].
This material was based upon work supported by an NSF Career Award to K. P. G. (CTS-9623450).

*Electronic address: giapis@ @ cheme.caltech.edu

[1] J. Proud et al., Plasma Processing of Materials: Scientific Opportunities and Technological Challenges (National Academy Press, Washington, DC, 1991).

[2] S. G. Ingram, J. Appl. Phys. 68, 500 (1990); J. C. Arnold and H. H. Sawin, ibid. 70, 5314 (1991).

[3] J. Liu et al., J. Appl. Phys. 68, 3916 (1990), and references therein.

[4] K. Hashimoto, Jpn. J. Appl. Phys. 32, 6109 (1993); 33, 6013 (1994).

[5] T. Nozawa et al., Jpn. J. Appl. Phys. 34, 2107 (1995).

[6] N. Fujiwara, T. Maruyama, and M. Yoneda, Jpn. J. Appl. Phys. 34, 2095 (1995); 35, 2450 (1996).

[7] T. Kinoshita, M. Hane, and J.P. McVittie, J. Vac. Sci. Technol. B 14, 560 (1996).

[8] G. S. Hwang and K.P. Giapis, J. Vac. Sci. Technol. B 15, 70 (1997).

[9] S. Samukawa and T. Tsukada, Appl. Phys. Lett. 69, 1056 (1996).

[10] T. Kinoshita, M. Hane, and J. P. McVittie, in 11th Plasma Processing Symposium Proceedings (The Electrochemical Society, New York, 1996).

[11] M. A. Lieberman and A. J. Lichtenberg, Principles of Plasma Discharges and Materials Processing (John Wiley \& Sons, Inc., New York, 1994).

[12] D. Vender and R. W. Boswell, IEEE Trans. Plasma Sci. 18, 725 (1990).

[13] For a self-consistent treatment of the sheath, $V_{\mathrm{dc}}$ could be determined from balancing the ion and electron currents to the wafer over an $\mathrm{rf}$ period. For $T_{e}=8 \mathrm{eV}$, this method yields $V_{\mathrm{dc}}=17 \mathrm{~V}$, i.e., very close to the value used in the simulation; here, the experimental value of $V_{\mathrm{dc}}=7 \mathrm{~V}$ for $T_{e}=4 \mathrm{eV}$ must also be used. Repeating the charging simulation for values of $V_{\mathrm{dc}}$ between 7 and $17 \mathrm{~V}$ produced slightly larger charging potentials for the poly-Si lines and the distribution along the trench bottom. Remarkably, the final sidewall etch profiles for $V_{\mathrm{dc}}=7$ and $14 \mathrm{~V}$ were virtually identical, indicative of a robust calculation capable of capturing the trends, despite the uncertainty in determining $V_{\mathrm{dc}}$.

[14] The "final overetch" step is simulated, referring to prolonged etching after the poly-Si on unpatterned areas is cleared and the trench bottoms become disconnected.

[15] M.S. Barnes, J.C. Foster, and J.H. Keller, IEEE Trans. Plasma Sci. 19, 240 (1991).

[16] T. Kamata and H. Arimoto, J. Appl. Phys. 80, 2637 (1996).

[17] Only specular reflection is considered; the energy transfer obeys hard-sphere collision kinematics with a gas-atom to surface mass ratio of 1.0; see also G.S. Hwang et al., Phys. Rev. Lett. 77, 3049 (1996).

[18] $100 \%$ overetching is excessive but was chosen to make the differences in the simulated profiles more dramatic. 